\title{
Accuracy of Postcontrast 3D Turbo Spin-Echo MR Sequence for the Detection of Enhanced Inflammatory Lesions in Patients with Multiple Sclerosis
}

\author{
J. Hodel, O. Outteryck, E. Ryo, A.-L. Bocher, O. Lambert, D. Chéchin, H. Zéphir, A. Lacour, J.-P. Pruvo, P. Vermersch, and X. Leclerc
}

\begin{abstract}
BACKGROUND AND PURPOSE: Therapeutic strategies for patients with MS partly rely on contrast-enhanced MR imaging. Our aim was to assess the diagnostic performance of 3D turbo spin-echo MR imaging with variable refocusing flip angles at 3T for the detection of enhanced inflammatory lesions in patients with multiple sclerosis.
\end{abstract}

MATERIALS AND METHODS: Fifty-six patients with MS were prospectively investigated by using postcontrast T-weighted axial 2D spin-echo and 3D TSE MR images. The order in which both sequences were performed was randomized. Axial reformats from 3D TT TSE were generated to match the 2D spin-echo images. The reference standard was defined by using clinical data and all MR images available. Three separate sets of MR images (2D spin-echo images, axial reformats, and multiplanar images from 3D TSE sequences) were examined in a blinded fashion by 2 neuroradiologists separately for the detection of enhanced MS lesions. Image artifacts and contrast were evaluated.

RESULTS: No artifacts related to vascular pulsation were observed on 3D TSE images, whereas image artifacts were demonstrated on 2D spin-echo images in 41 patients. One hundred twelve enhanced MS lesions were identified in 19 patients. Sixty-four lesions were correctly diagnosed by using 2D spin-echo images; 90 , by using 3D TSE axial reformatted views; and 106, by using multiplanar analysis of the 3D TSE sequence. Multiplanar analysis was $94.7 \%$ sensitive and $100 \%$ specific for the diagnosis of patients with at least 1 enhanced lesion. Contrast of enhanced MS lesions was significantly improved by using the 3D TSE sequence $(P<.011)$.

CONCLUSIONS: The 3D TSE sequence with multiplanar analysis is a useful tool for the detection of enhanced MS lesions.

ABBREVIATIONS: $C R=$ contrast rate; $\mathrm{DIR}=$ double inversion recovery; $\mathrm{GRE}=$ gradient recalled-echo; $\mathrm{SE}=$ spin-echo

$\mathbf{T}$ he diagnosis of multiple sclerosis needs to demonstrate dissemination of brain lesions in space and time and to exclude alternative diagnoses. In some circumstances, dissemination of brain lesions in space and time can be established by a single MR imaging. ${ }^{1}$ In such patients, dissemination of brain lesions in time is demonstrated by the simultaneous presence of asymptomatic gadolinium-enhancing and nonenhancing lesions at any time. ${ }^{1}$ Indeed, therapeutic strategies for patients with MS partly rely on contrast-enhanced MR imaging. Updated recommendations on the use of MR imaging in MS suggest that axial 2D T1-weighted spin-echo (SE) MR imaging should be performed for the detection of enhanced MS lesions, ${ }^{2,3}$ whereas T1-weighted 3D gradient

Received May 7, 2013; accepted after revision July 2.

From the Departments of Neuroradiology (J.H., E.R., A.-L.B., J.-P.P., X.L.) and Neurology (O.O., H.Z., A.L., P.V.), Hôpital Roger Salengro, Lille, France; Department of Neuroradiology (O.L.), Fondation Ophtalmologique Rothschild, Paris, France; and Philips Medical Systems (D.C.), Suresnes, France.

Please address correspondence to Jérôme Hodel, MD, Department of Neuroradiology, Hôpital Roger Salengro, Rue Emile Laine 59037 Lille, France; e-mail: jerome.hodel@gmail.com

http://dx.doi.org/10.3174/ajnr.A3795 recalled-echo (GRE) MR images are reported to be useful for the assessment of brain atrophy. ${ }^{2-4}$ As previously reported, ${ }^{5}$ the sensitivity of the 2D T1-weighted SE sequence for the detection of enhanced MS lesions is progressively increased from 5 to $10 \mathrm{~min}$ utes after intravenous injection of gadolinium.

Recently, a new technique has been introduced to generate 3D T1-weighted images of the brain (BrainView, Philips Healthcare, Best, the Netherlands; Cube, GE Healthcare, Milwaukee, Wisconsin; SPACE, Siemens, Erlangen, Germany). The BrainView sequence is based on a turbo spin-echo acquisition with variable refocusing flip angles and short echo spacing, allowing longer echo-train readouts and reduced signal losses. ${ }^{6}$ Such an approach provides high spatial resolution and signal-to-noise ratio without the blurring commonly associated with long echo-trains. Thinner section images can be acquired, minimizing the partial volume effect between small lesions and surrounding brain parenchyma. The added value of the postcontrast 3D TSE sequence with variable flip angles was recently reported in patients with brain metastasis. $^{7,8}$

No data are available on the diagnostic performance of the 


\begin{tabular}{|c|c|c|c|c|}
\hline Parameter & 3D DIR & 2D FLAIR & 2D TI SE & 3D TI TSE \\
\hline TR (ms) & 5500 & 11,000 & $600-700$ & 400 \\
\hline $\mathrm{TE}(\mathrm{ms})$ & 251 & 125 & 10 & 26 \\
\hline $\mathrm{TI}(\mathrm{ms})$ & $625-2600$ & 2800 & N/A & $\mathrm{N} / \mathrm{A}$ \\
\hline Echo-train length & 173 & 31 & N/A & 30 \\
\hline No. of sections/slab & 300 & 31 & 36 & 267 \\
\hline Section thickness (mm) & 1.3 & 5 & 4 & 1.2 (reformat 4) \\
\hline Matrix size & $208 \times 208$ & $276 \times 126$ & $232 \times 165$ & $256 \times 215$ \\
\hline Voxel size (mm) & $1.2 \times 1.2 \times 1.3$ & $0.85 \times 1.42 \times 5$ & $1 \times 1.10 \times 4$ & $1 \times 1.10 \times 1.2($ reformat $1 \times 1.10 \times 4)$ \\
\hline $\mathrm{FOV}(\mathrm{mm})$ & $250 \times 250$ & $230 \times 176$ & $230 \times 182$ & $250 \times 237$ \\
\hline Acquisition plane & Sagittal & Axial & Axial & Sagittal \\
\hline Acquisition time & $6 \mathrm{~min} 30 \mathrm{sec}$ & $2 \min 56 \mathrm{sec}$ & $3 \mathrm{~min} 30 \mathrm{sec}$ & $5 \mathrm{~min} 30 \mathrm{sec}$ \\
\hline
\end{tabular}

Note:-N/A indicates not applicable; No. of sections, number of sections acquired; reformat, 4-mm-thick axial reformatted views of the 3D T1 TSE sequence; 2D, TI SE images and 4-mm-thick axial reformatted views of the 3D TI TSE sequence with the same voxel size.

postcontrast 3D T1-weighted TSE sequence for the detection of enhanced inflammatory lesions in patients with MS. Our purpose was to assess the detectable enhanced MS lesions by using the 3D BrainView sequence compared with the conventional axial 2D SE sequence.

\section{MATERIALS AND METHODS \\ Patients}

This study was approved by our institutional review board. Informed consent was obtained from all patients. From July to December 2012, fifty-nine consecutive patients with MS according to the $2010 \mathrm{McD}$ onald criteria were examined by using 3T MR imaging for follow-up. Three of these 59 patients were excluded because of motion artifacts. Finally, 56 consecutive patients (20 men, 36 women; mean age, $41.2 \pm 13.9$ years) were included in the study.

\section{Image Acquisition}

All patients were examined on a $3 \mathrm{~T}$ MR imaging scanner (Achieva; Philips Healthcare) by using a 32-channel array head coil. The routine MR imaging protocol included 2D axial MR images (pre- and postcontrast T1 SE, diffusion-weighted T2 TSE, and FLAIR) and 3D double inversion recovery (DIR) MR images. In addition, the postcontrast 3D TSE T1-weighted sequence (3D BrainView) was performed in all patients. The 3D TSE sequence is characterized by a long TSE impulsion train and a combination of advanced features, among which are variable refocusing angles. A specific refocalization scheme is also applied to preserve $\mathrm{T} 1$ contrast and minimize the blurring effect. Intravenous gadobutrol (Gadovist 1.0; Bayer-Schering Pharma, Berlin, Germany) was administered at a concentration of $0.1 \mathrm{mmol} / \mathrm{kg}$. The 2D axial SE and 3D TSE T1-weighted postcontrast MR images were obtained 10 minutes after the gadolinium administration for all patients. The order in which these 2 postcontrast sequences were performed was randomized. 3D TSE source images were reformatted into 4-mm-thick axial sections to match the section thickness and the acquisition plane of the 2D SE T1-weighted images.

The parameters for each MR image are summarized in the Table.

\section{Image Analysis}

The reference standard for the diagnosis of enhanced MS lesions was defined by 2 neuroradiologists (X.L. and J.-P.P.) with $>25$ years' experience. They reviewed together all the MR imaging datasets by using clinical data and all the MR images available. An enhanced MS lesion was defined as a focal enhancement on postcontrast images, with a size superior to $2 \mathrm{~mm}$, involving gray or white matter and associated with a parenchymal hyperintensity on FLAIR or DIR images. Focal enhancement without DIR- or FLAIR-associated hyperintensities was not considered a real MS lesion. First, the readers were asked to assess enhanced MS lesions for each patient. Second, the readers were asked to notice highsignal-intensity artifacts related to vascular pulsation within the postcontrast 2D SE and 3D TSE images according to the following scale: 0 , no artifacts; 1 , artifacts that did not impair image analysis; 2 , artifacts that impaired image analysis.

For the evaluation of the 3D TSE sequence, 2 neuroradiologists (E.R. and A.-L.B.) with 7 and 6 years' experience, respectively, analyzed, independently and separately, all postcontrast T1-weighted images on a PACS workstation. First, the readers assessed 3 sets of images in a randomized order: 1) 2D axial SE images, 2) 4-mm-thick axial reformations from 3D TSE sequences, and 3) multiplanar analysis from 3D TSE sequences with thin sections and MIP reformations in arbitrary planes. They were blinded to clinical data and to other MR images and were asked to notice the number and location of the enhanced inflammatory lesions for each patient. An enhanced lesion was retained when a focal enhancement involving the gray or white matter was detected. Second, a consensus reading was performed between the 2 blinded readers to resolve their disagreements.

One radiologist (X.L.) measured the contrast rate (CR) of the largest enhanced MS lesions (ie, measuring at least $1 \mathrm{~cm}$ ) by using both the axial 2D T1 SE images (4-mm-thick) and the 4-mmthick axial reformatted views from the 3D TSE sequence. For each patient, regions of interest were drawn within the enhanced MS lesions and their background (ie, adjacent white matter), allowing the measurement of signal intensities (SI). Using the PACS workstation, we drew the same ROIs on the $2 \mathrm{D}$ images and axial reformatted views. As previously reported, ${ }^{8,9}$ the contrast rate was calculated between the MS lesion and its background according to the following formula: $\mathrm{CR}=[($ SI Lesion - SI Background $) / S I$ Background] $\times 100$.

\section{Statistical Analysis}

All statistical analyses were performed by using R Statistical Software (Version 3.0.0; http://www.r-project.org/). 


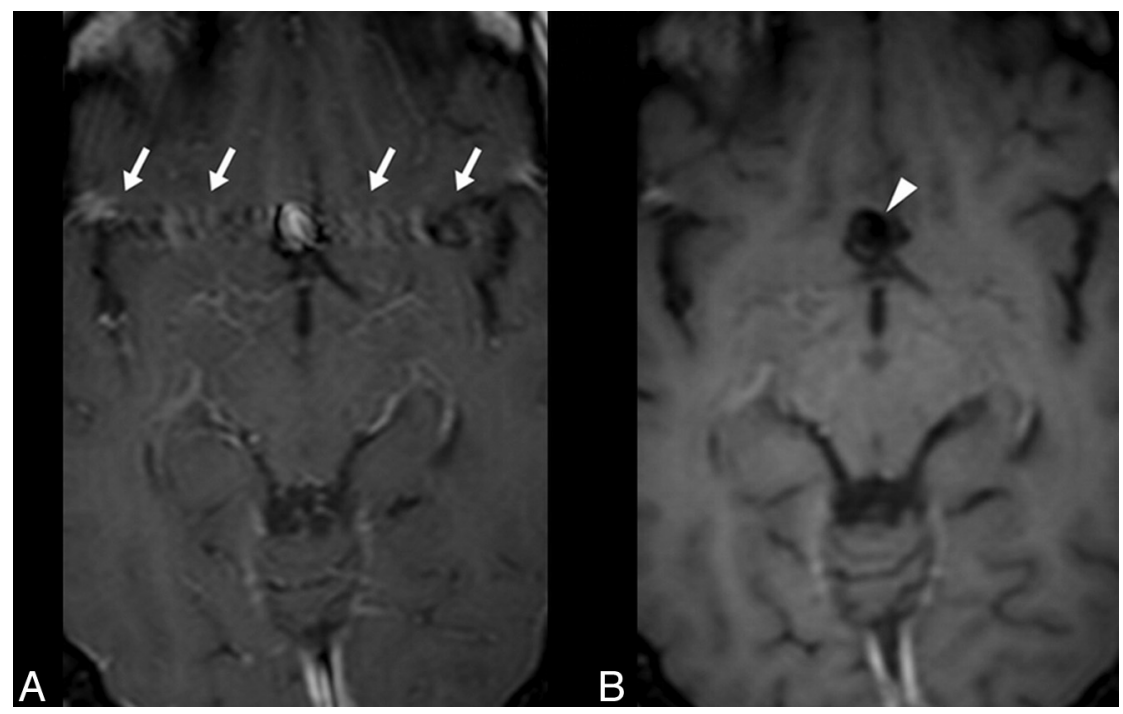

FIG 1. Postcontrast T1-weighted 2D SE $(A)$ and a 4-mm-thick axial reformatted view of the 3D TSE $(B)$ images of patient 5 with MS and an aneurysm of the anterior communicating artery. High-signal-intensity artifacts related to vascular pulsation are observed beside the aneurysm ( $A$, arrows) by using axial 2D SE images, while these artifacts are not visible on 3D TSE images ( $B$, arrowhead). Similar differences can be noted between the 2 postcontrast sequences within the brain sinuses. Indeed, while the 2D SE sequence is still influenced by blood flow effects, a black-blood effect is induced by the long echo-trains used with the 3D TSE sequence ( $B$, arrowhead). This technical aspect may also partly explain the improved diagnostic performance observed with the 3D TSE sequence.

Interobserver agreement was calculated by using the Cohen $\kappa$ test. $\kappa$ values were interpreted as follows: $\kappa=0$ indicates poor agreement; $\kappa=0.01-0.20$, minor agreement; $\kappa=0.21-0.40$, fair agreement; $\kappa=0.41-0.60$, moderate agreement; $\kappa=0.61-0.80$, good agreement; and $\kappa=0.81-1$, excellent agreement.

To compare 2D SE and 3D TSE sequences, we evaluated both the overall number of enhanced MS lesions detected and the number of patients diagnosed with at least 1 enhanced MS lesion. The overall number of enhanced MS lesions detected by using 2D axial SE images, 4-mm-thick axial reformations from 3D TSE sequences, and multiplanar analysis of the 3D TSE sequences was compared with the reference standard by using the Cohen $\kappa$ test. For the diagnosis of patients with at least 1 enhanced MS lesion, standard diagnostic accuracy parameters (sensitivity and specificity) of 2D axial SE images, 4-mm-thick axial reformations from the 3D TSE sequences, and multiplanar analysis of the 3D TSE sequences were calculated according to the reference standard.

Comparisons of $\kappa$ values were performed to determine whether the agreements were significantly different for each parameter evaluated.

We also compared the MS lesion contrast rate and the artifacts grading between 2D SE and 3D TSE sequences by using a nonparametric statistical test (Wilcoxon test). $P$ values $<.05$ were considered statistically significant.

\section{RESULTS}

\section{Overall Image Quality}

High-signal-intensity artifacts related to vascular pulsation were observed in 41 of the 56 included patients by using the 2D T1 sequence involving the posterior fossa $(n=38)$ or the frontal lobes $(n=8)$ (median score $=1)$. In comparison with the 2D SE sequence, there were no artifacts related to vascular pulsation by using the 3D TSE sequence $(P<.001)$. A slight blurring was reported for 15 patients on 3D TSE images, which did not impair the data analysis.

Figure 1 illustrates the reduction of artifacts by using the 3D TSE sequence.

\section{Reference Standard for the Diagnosis of Enhanced MS Lesions}

Using clinical data and all the MR images available, we diagnosed 112 MS lesions in 19 patients; 9 lesions involved the gray matter while 103 lesions involved the periventricular or deep white matter. Six of the 112 enhanced lesions were only confirmed with the DIR images and were not visible on conventional 2D FLAIR images.

\section{Interobserver Agreement}

For the detection of enhanced MS lesions, interobserver agreement was substantial for $2 \mathrm{D}$ axial SE images $(\kappa=0.62)$ and good for 3D TSE images $(\kappa=0.75)$. Comparison of the $\kappa$ values showed that the interobserver agreement was not significantly different between 2D axial SE and 3D TSE images $(P=$ .2140)

\section{Comparison of 2D TI SE and 3D TSE MR Images}

Compared with the reference standard, reader 1 correctly identified $57.1 \%$ of the enhanced MS lesions by using the 2D T1 SE sequence, $77.68 \%$ by using 4 -mm-thick axial reformatted views, and $93.8 \%$ by using the multiplanar analysis. Reader 2 identified $56.3 \%, 79.5 \%$, and $93.8 \%$ of the enhanced MS lesions, respectively. No enhanced MS lesion was visible only on 2D T1weighted SE images.

Using the 2D SE and 3D TSE MR images, the 2 blinded readers misdiagnosed a total of 22 focal venous enhancements as enhanced MS lesions (ie, 22 false-positive lesions because these lesions were not previously considered real MS lesions according to the reference standard). Twelve false-positive lesions were observed by using the 2D T1-weighted SE sequence, 7 by using 4-mm-thick axial reformatted views, and 3 by using the multiplanar analysis of the 3D TSE sequence.

The agreement with the reference standard was fair by using the 2D T1 SE images $(\kappa=0.284)$, moderate by using $4-\mathrm{mm}$-thick axial reformatted views $(\kappa=0.507)$, and good by using the multiplanar analysis $(\kappa=0.735)$. Agreement with the reference standard was significantly improved by using the multiplanar analysis of the 3D TSE sequence compared with 2D T1 SE images $(P=$ $.0007)$ or 4 -mm-thick axial reformatted views $(P=.0465)$.

On the basis of consensus statements between the blinded readers, 64 enhanced MS lesions were correctly identified by using the 2D T1-weighted SE sequence. Forty-two additional lesions were visible by using the 3D TSE sequence; 26 lesions, on 4-mmthick axial reformatted views (90 lesions detected); and 16 other 


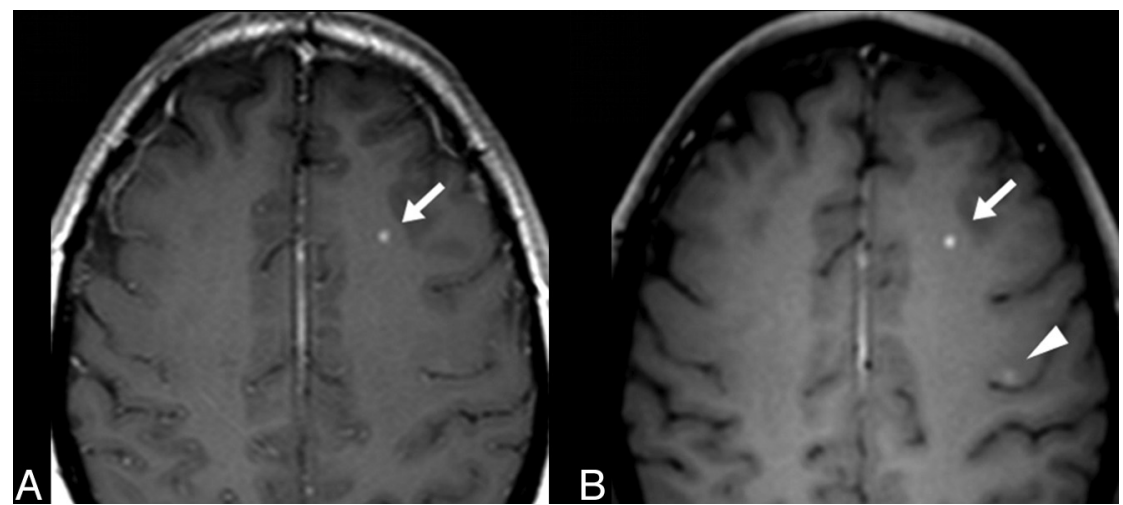

FIG 2. Postcontrast T7-weighted 2D SE $(A)$ and 4-mm-thick axial reformatted views of the 3D TSE (B) images of patient 24 with MS. An active enhanced MS lesion is detected within the left frontal white matter by using both axial 2D SE images (A, arrow) and a 4-mm-thick axial reformatted view of the 3D TSE sequence ( $B$, arrow). Due to improved image contrast, an additional lesion involving the precentral sulcus is visible by using the 3D TSE sequence ( $B$, arrowhead).
To improve the comparison between 2D SE and 3D TSE postcontrast MR images, we first used 4-mm-thick axial reformations from the 3D TSE sequence leading to the same voxel size. This approach confirmed that the diagnostic performance of the 3D TSE sequence was significantly superior to that of the 2D T1 SE. This difference could be partly explained by the suppression of artifacts related to vascular pulsation. As previously suggested, ${ }^{10}$ the black-blood effect of the 3D TSE sequence is due to the dephasing of spins induced by the long echo-trains used. In our study, we systematically observed this effect within brain arteries, while a discontinuous enhancement was still observed within large cortical veins. lesions, by using the multiplanar analysis (106 lesions detected). The 26 additional lesions revealed by the axial reformatted views involved the periventricular white matter $(n=12)$, the optic nerves $(n=2)$, the pons $(n=5)$, the cerebellum $(n=4)$, and the temporal $(n=2)$ and the frontal $(n=1)$ white matter. The 16 additional lesions detected by multiplanar analysis involved the periventricular white matter $(n=6)$, the corpus callosum $(n=3)$, the cingulum $(n=1)$, the brain stem $(n=4)$, the parietal white matter $(n=1)$, and the optic nerve $(n=1)$.

Sixteen of the 56 patients presented with at least 1 enhanced MS lesion with 2D SE images; 17, with axial reformatted views; and 19, with multiplanar analysis of the 3D TSE sequences. Indeed 3 patients were correctly diagnosed with at least 1 MS enhanced lesion only with the 3D TSE sequence. Such findings had an impact on clinical management by switching on second-line immunosuppressive therapy. For the diagnosis of the patients with at least $1 \mathrm{MS}$ enhanced lesion, 2D axial SE images were $84.2 \%$ sensitive and $78.4 \%$ specific, $4-\mathrm{mm}$-thick axial reformations of the 3D TSE sequences were $84.2 \%$ sensitive and $89.2 \%$ specific, and multiplanar analysis of the 3D TSE sequences was $94.7 \%$ sensitive and $100 \%$ specific.

\section{Contrast Rate}

ROIs were drawn within MS active lesions that measured at least 1 cm (11 patients). With 2D SE images, the median CR was 39.5 (11.9; 100.3), while, with 3D TSE images, the median CR was 46.9 $(10.3 ; 128)$. The CR of the enhanced MS lesions was significantly higher by using the 3D TSE sequence $(P<.011)$.

An example of the improved detectability of enhanced MS lesions by using the 3D TSE sequence is given in Fig 2 .

\section{DISCUSSION}

In this study, we demonstrated that the 3D TSE sequence improved the detection of enhanced MS lesions due to a better image contrast and the suppression of artifacts related to vascular pulsation. The use of thinner sections and MIP reformations further improved the diagnosis of small MS lesions. These findings are clinically relevant, considering the importance of enhanced asymptomatic lesions for the diagnosis and management of patients with MS.
The non-spatially selective pulse, which affects the entire imaging slab by using the 3D TSE sequence, may also contribute to the absence of artifacts related to vascular pulsation. ${ }^{6}$ In our study, such technical aspects were particularly useful for the detection of MS lesions involving the brain stem or the cerebellum.

The diagnostic performance of the 3D TSE sequence was further improved when using thinner sections and multiplanar analysis. There are several advantages of using $3 \mathrm{D}$ thinner sections for the detection of enhanced MS lesions. First, it has been previously reported that the decrease in section thickness (ie, of the partial volume effect) is strongly associated with increased lesion detection. ${ }^{11-13}$ Second, 3D images can be registered more accurately, allowing a more precise assessment of spatiotemporal disease activity that is crucial in patients with MS. In our study, registration of MR images was also useful to define the reference standard. Indeed, the 3D DIR images were registered with the 3D T1 TSE images, optimizing the detectability of small enhanced MS lesions. ${ }^{14-16}$ Third, 3D sequences, with their higher SNR and isotropic voxel size, allow image postprocessing with MIP reformations in arbitrary planes. MIP views can be routinely obtained with the 3D TSE sequence because the contrast between gray and white matter appears significantly reduced. This is not the case when using 3D T1-weighted GRE MR images. We may hypothesize that MIP reformatted views could improve the time efficiency for the detection of enhanced MS lesions. Further studies including a larger cohort of patients with enhanced MS lesions may confirm this hypothesis.

Our study has several limitations. We used the DIR sequence, which is not a standard pulse sequence for clinical use. The DIR sequence may have further improved the detection of cortical and brain stem MS lesions as previously reported. ${ }^{14-16}$ However, the relatively small number of enhanced MS lesions confirmed only with DIR images should not limit the generalizability of our findings to clinical practice. The parameter setting of the 3D TSE sequence in the present study was optimized before scanning patients to decrease image artifacts and to improve the image contrast. An acquisition time of more than 20 minutes would be required to achieve a full brain coverage with the same section thickness for both axial SE and 3D TSE sequences (ie, $1.2 \mathrm{~mm}$ ). 
Then, the use of axial 4-mm-thick reformatting views of the BrainView sequence, which perfectly matched the axial T1 SE images, appeared acceptable to achieve an equivalent comparison between the 2 postcontrast MR images.

Using the 3D TSE sequence, there was a slightly increased scanning time compared with the 2D SE sequence. The duration of the 3D TSE sequence could be reduced by applying a higher parallel imaging acceleration factor. Moreover, in most centers, the MR imaging protocol usually includes 2 postcontrast T1weighted 2D SE sequences in the coronal and axial planes for routine evaluation. Image blurring may be observed on 3D TSE images due to the longer echo-trains. However, this did not prevent image interpretation, and a specific refocalization scheme was developed to minimize these artifacts. Finally, discontinuous enhancement within large cortical veins may be misinterpreted as an enhanced MS lesion on 3D TSE images. In our study, due to the high spatial resolution of this sequence, multiplanar reformations were particularly useful to distinguish these veins from brain MS lesions.

\section{CONCLUSIONS}

This study suggests that the 3D T1-weighted TSE MR imaging with variable refocusing flip angles is superior to the conventional 2D SE axial sequence for the detection of enhanced MS lesions. In addition, multiplanar analysis with thinner sections and MIP reformatted views further improves the diagnostic performance of the 3D TSE sequence.

\section{ACKNOWLEDGMENTS}

Level of contributions: Jérôme Hodel: data acquisition and analysis, manuscript editing; Olivier Outteryck and Xavier Leclerc: data analysis, manuscript editing; Edouard Ryo, Anne-Laure Bocher, and Jean-Pierre Pruvo: data acquisition and analysis; David Chéchin: data analysis; Patrick Vermersch, Hélène Zéphir, Arnaud Lacour: data acquisition; and O. Lambert: statistical analysis.

Disclosures: Oliver Outteryck—UNRELATED: Travel/Accommodations/Meeting Expenses Unrelated to Activities Listed: Bayer-Schering, , Biogen Idec, ${ }^{*}$ Teva Pharmaceuticals, ${ }^{*}$ Merck-Serono,* Novartis. David Chéchin-OTHER RELATIONSHIPS: employment at Philips Healthcare, Comments: I am employed by Philips Healthcare, and this work was done on a Philips 3T MRI system. I supported this work by installing, evaluating, and optimizing the sequences. Hélène Zéphir-UNRELATED: Consultancy: Biogen Idec, Genzyme, Sanofi, Merck, Teva, Novartis, Bayer, Grants/Grants Pending: Teva.* Patrick Vermersch-UNRELATED: Consultancy: Biogen Idec, Novartis, Bayer, Merck-Serono, Teva, Sanofi-Genzyme, Grants/Grants Pending: Biogen

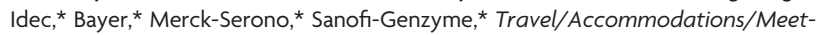
ing Expenses Unrelated to Activities Listed: Biogen Idec, Novartis, Bayer, MerckSerono, Teva, Sanofi-Genzyme. *Money paid to the institution.

\section{REFERENCES}

1. Polman CH, Reingold SC, Banwell B, et al. Diagnostic criteria for multiple sclerosis: 2010 revisions to the McDonald criteria. Ann Neurol 2011;69:292-302

2. Lövblad KO, Anzalone N, Dorfler A, et al. MR imaging in multiple sclerosis: review and recommendations for current practice. AJNR Am J Neuroradiol 2010;31:983-89

3. Filippi M, Rocca MA. MR imaging of multiple sclerosis. Radiology 2011;259:659-81

4. Bergsland N, Horakova D, Dwyer MG, et al. Subcortical and cortical gray matter atrophy in a large sample of patients with clinically isolated syndrome and early relapsing-remitting multiple sclerosis. AJNR Am J Neuroradiol 2012;33:1573-78

5. Uysal E, Erturk SM, Yildirim H, et al. Sensitivity of immediate and delayed gadolinium-enhanced MRI after injection of $0.5 \mathrm{M}$ and 1.0 $\mathrm{M}$ gadolinium chelates for detecting multiple sclerosis lesions. $A J R$ Am J Roentgenol 2007;188:697-702

6. Mugler JP 3rd, Bao S, Mulkern RV, et al. Optimized single-slab three-dimensional spin-echo MR imaging of the brain. Radiology 2000;216:891-99

7. Reichert M, Morelli JN, Runge VM, et al. Contrast-enhanced 3-dimensional SPACE versus MP-RAGE for the detection of brain metastases: considerations with a 32-channel head coil. Invest Radiol 2013;48:55-60

8. Kato $\mathrm{Y}$, Higano $\mathrm{S}$, Tamura $\mathrm{H}$, et al. Usefulness of contrast-enhanced T1-weighted sampling perfection with application-optimized contrasts by using different flip angle evolutions in detection of small brain metastasis at 3T MR imaging: comparison with magnetization-prepared rapid acquisition of gradient echo imaging. AJNR Am J Neuroradiol 2009;30:923-29

9. Rand S, Maravilla KR, Schmiedl U. Lesion enhancement in radiofrequency spoiled gradient-echo imaging: theory, experimental evaluation, and clinical implications. AJNR Am J Neuroradiol 1994;15:27-35

10. Busse RF, Brau AC, Vu A, et al. Effects of refocusing flip angle modulation and view ordering in 3D fast spin echo. Magn Reson Med 2008;60:640-49

11. Dolezal O, Dwyer MG, Horakova D, et al. Detection of cortical lesions is dependent on choice of slice thickness in patients with multiple sclerosis. Int Rev Neurobiol 2007;79:475-89

12. Molyneux PD, Tubridy N, Parker GJ, et al. The effect of section thickness on MR lesion detection and quantification in multiple sclerosis. AJNR Am J Neuroradiol 1998;19:1715-20

13. Bink A, Schmitt M, Gaa J, et al. Detection of lesions in multiple sclerosis by 2D FLAIR and single-slab 3D FLAIR sequences at $3.0 \mathrm{~T}$ : initial results. Eur Radiol 2006;16:1104-10

14. Moraal B, Roosendaal SD, Pouwels PJ, et al. Multi-contrast, isotropic, single-slab 3D MR imaging in multiple sclerosis. Eur Radiol 2008; $18: 2311-20$

15. Simon B, Schmidt S, Lukas C, et al. Improved in vivo detection of cortical lesions in multiple sclerosis using double inversion recovery MR imaging at 3 Tesla. Eur Radiol 2010;20:1675-83

16. Geurts JJ, Pouwels PJ, Uitdehaag BM, et al. Intracortical lesions in multiple sclerosis: improved detection with 3D double inversionrecovery MR imaging. Radiology 2005;236:254-60 